\title{
Robustness of Three-Phase Equilibrium Calculations
}

\author{
S. E. Gorucu, Computer Modelling Group, segorucu@gmail.com (Corresponding author) \\ R. T. Johns, The Pennsylvania State University, rjohns@psu.edu
}

\begin{abstract}
Phase equilibrium calculations converge less often as the number of phases and components increase, and for overall compositions closer to phase split boundaries and critical points. Computational speed and robustness of flash calculations are very important aspects for pipeline transmission and for reservoir simulation where billions of flash calculations may be done. Reduced methods have the potential to improve the robustness of phase equilibrium calculations because if chosen properly they can linearize the equations, use fewer independent variables and can be unbounded. Improved robustness could further improve the speed and accuracy of compositional simulation and avoid false two-phase solutions, where three or more phases may be present.

In this paper, we use the reduced variables of Gorucu and Johns (2014) to test robustness in performing two- and three-phase stability analysis and corresponding flash calculations. These multiphase equilibrium calculations are compared with the conventional phase equilibrium calculations based on minimization of Gibbs energy and the reduced method proposed by Okuno et. al. (2010a). Using thousands of equally-spaced and unbiased multi-phase equilibrium calculations, the proposed multi-phase equilibrium calculations are shown to be more robust than the other two tested algorithms.
\end{abstract}

\section{Introduction}

Petroleum processes such as gas flooding or condensate gas/volatile oil production require compositional modeling where the movement of chemical components between the phases and in the reservoir must be tracked. A typical oil reservoir consists of thousands of hydrocarbon and nonhydrocarbon components that are lumped into few components and pseudocomponents. Although desirable for improved accuracy, simulation using many components is not practical with standard flash calculation procedures owing to large computational time. Current practice is to limit the number of defined and undefined components to between six and fifteen depending on how many heavy pseudocomponents (undefined components) are desired. Further, nearly all reservoir simulators do not perform three-phase flash calculations owing to their slow computational time, and increased likelihood of nonconvergence. The accuracy of reservoir simulations, however, decrease as more heavy components are lumped and/or as potential equilibrium phases are neglected.

There are likely an optimum number of components to use in simulation depending on computational speed, and the needed accuracy of the fluid modeled. Surface facility design for example generally requires a detailed fluid description of between 20 and 30 components. It would be desirable then to use 20 or more components to match those requirements, and to reduce errors in reservoir simulation associated with pseudocomponent lumping and neglected additional equilibrium phases. Reduced variables in phase behavior calculations are one possible approach to model more components and multiple phases in equilibrium more efficiently and robustly.

Phase equilibrium calculations become slower not only due to an increased number of components but also to an increased number of phases. The occurrence of a third hydrocarbon phase is common during low temperature gas injection, e.g. $\mathrm{CO}_{2}$ injection in West Texas at temperatures less than 
about $100^{\circ} \mathrm{F}$ where a $\mathrm{CO}_{2}$-rich liquid phase, hydrocarbon-rich liquid phase and a vapor phase may appear (Turek et al., 1988), and also for injection of viscosity-reducing solvent in heavy oil reservoirs (Li et. al., 2013). Calculations with an aqueous phase can also be important, adding the potential of four phases in equilibrium in some Permian basin $\mathrm{CO}_{2}$ floods (Mohebbinia et al., 2013). Three-phase equilibrium calculations may also be important for $\mathrm{CO}_{2}$ sequestration in oil reservoirs where the amount of $\mathrm{CO}_{2}$ in the brine is of interest. Asphaltenes can also form during injection of volatile components so that five phases may coexist (Mohebbinia et al., 2014).

Phase equilibrium calculations usually consist of two basic steps: stability analysis and flash calculations. Stability analysis is used to save computational time by determining the number of stable equilibrium phases. Flash calculations then compute equilibrium phase compositions and phase mole fractions if stability analysis shows that a phase split should occur. Typically, phase equilibrium calculations begin by assuming a single phase exists in a given grid block and time step. If that phase is found unstable by stability analysis then two-phase flash calculations are carried out. A further check of stability is made on one of the two equilibrium phases. If unstable, a three-phase flash calculation should be done, although current practice is not to do this calculation because of increased computational time requirements and robustness of the calculations. Avoiding these calculations can result in significant inaccuracies in predicted recoveries (Okuno et. al., 2010b). It would be advantageous therefore to have a robust and fast approach to three-phase (or more) equilibrium calculations.

Reduced methods can avoid excessive computational times when multiple phases and components are present. Previous research has proposed and analyzed reduced methods of various types (Michelsen, 1986; Hendriks, 1988; Hendriks \& Van Bergen, 1992; Jensen \& Fredenslund, 1993; Li \& Johns, 2006; Nichita et al., 2006; Okuno et al., 2010a; Nichita \& Graciaa, 2011; Petitfrere \& Nichita, 2015a). All of these aforementioned authors have focused on the computational speed or number of iterations of the reduced methods, and generally on only two-phase reduced methods. The robustness of two-phase flash calculations and stability analysis is critical to subsequent three-phase or even to fourphase flash calculations as the converged two-phase calculations are the initial input for subsequent multiphase flash calculations.

While many reduced methods have been proposed and their computational speed improvements have been investigated, there has been very little research on the potential improved robustness of the reduced methods (Pan \& Firoozabadi, 2002; Okuno, 2009; Gorucu and Johns, 2014, 2015). Pan and Firoozabadi (2002) analyzed the tangent plane distance (TPD) for an example fluid and concluded that the reduced method had only one minimum in reduced space, while the conventional technique in compositional space had two observable minima for the fluid tested. This conclusion has been questioned by Haugen and Beckner (2013) who stated that the reduced number of minima may be related to a small change in phase behavior owing to the reduced technique used (eigenvalue decomposition where small eigenvalues are set to zero). Okuno et al. (2009, 2010a), however, reported the number of failed flash calculations as the number of components increases and showed that the number of convergence failures were significantly larger with conventional techniques. In their reduced approach the phase behavior predictions were identical between the conventional and reduced methods so that the improvement in robustness was more certain, at least for the fluids studied. Recently, Petitfrere and Nichita (2015b) introduced a new multiphase equilibrium calculation technique using a reduction method which is very similar to our algorithm (Gorucu, 2013). In their paper, they also show that their reduction method is equivalent to an unbounded minimization problem. However, Petitfrere and Nichita implemented reduced parameters based on eigenvalue decomposition, which has been shown to be significantly slower than when the empirical BIP formula of Li and Johns (2006) is used (Gorucu and Johns 2015).

Because the reduced methods decrease the number of independent variables, the reduced methods could exhibit less complicated behavior compared to the conventional technique owing to significantly smaller search space (five-component space with Li and Johns (2006) reduced variables compared to $N_{C}$ hyperspace with conventional methods). Gorucu and Johns (2015) implemented published two-phase reduced and conventional flash calculations and compared these techniques in terms of speed and robustness. Their results for the numerous fluids studied showed that the reduced variables of Nichita and 
Graciaa (2011) were more robust compared to other reduced and conventional techniques likely because of the linearization of the fugacity expression. Gorucu and Johns results were based on thousands of unbiased two-phase flash calculations.

In this paper, we focus on the improvement in robustness of published three-phase reduced methods. First, we briefly describe our algorithm for conventional phase equilibrium calculations. Then, we implement the reduced variables of Gorucu and Johns (2014) for both stability analysis and threephase flash calculations. We then give our methodology to test and compare the various algorithms using several example fluids where three-phase regions co-exist. Finally, we compare our new reduced singlephase stability analysis, two-phase flash calculations, two-phase stability analysis and three-phase flash calculations with the results of other two techniques i.e.; the reduced method of Okuno et al. (2010a), and the conventional approach based on minimization of Gibbs energy (Perschke et. al., 1989, Michelsen \& Mollerup, 2007).

\section{Conventional Phase Equilibrium Calculations}

There are two common ways to perform conventional flash calculations. One method is based on equality of component chemical potentials (fugacities), while another is based on minimizing Gibbs energy. The later approach has been shown to be computationally more efficient and is the method used for conventional flash calculation in this paper.

Flash calculations normally are preceded by stability analysis, since stability analysis is not as computationally intensive. If stability analysis indicates a stable phase(s), then flash calculations are not performed. Stability analysis is based on the minimization of tangent plane distance. We used the stability analysis algorithm suggested by Michelsen and Mollerup (2007) for all calculations with the objective function,

$$
\operatorname{tm}(\boldsymbol{W})=1+\sum_{i=1}^{N_{C}} W_{i}\left(\ln W_{i}+\ln \varphi_{i}(\boldsymbol{W})-d_{i}-1\right)
$$

In Eq. (1), the independent variable is $2 \sqrt{W_{i}}$ where $W_{i}$ are compositional variables that are treated as mole numbers. Their expression allows cancelling a term from the Hessian matrix.

The objective function for the flash calculations is the dimensionless Gibbs energy $(G / R T)$ and the independent variables are the phase mole numbers. Therefore, the gradient vector is

$$
g_{i}=\ln f_{i}^{\beta}-\ln f_{i}^{1}
$$

where $\beta=2$ or 3 . We used the Hessian matrix approximation of Michelsen and Mollerup (2007) for stability analysis. More details on the derivations can be found at Perschke et. al. (1989) and Michelsen and Mollerup (2007). 


\section{Reduced Stability Analysis}

The reduced methods are based on expressing the fugacity coefficient in as few parameters as possible. Gorucu and Johns (2015) provide an extensive catalogue of the types of reduced methods and their derivations. In this section, we first provide a summary of a few reduced methods that provide a representative sampling of available methods, and then we introduce our new reduced parameters using a combination of the empirical BIP formula of Li and Johns (2006) and the approach of Nichita and Graciaa (2010).

The expression for the fugacity coefficient is

$$
\phi_{i}=\exp \left(\frac{B_{i}}{B}(Z-1)-\ln (Z-B)-\frac{A}{2 \sqrt{2 B}}\left(\frac{2 \sum_{j} x_{j} A_{i j}}{A}-\frac{B_{i}}{B}\right) \ln \left(\frac{Z+(1+\sqrt{2}) B}{Z+(1-\sqrt{2}) B}\right)\right)
$$

where $A=\sum_{i=1}^{N_{C}} \sum_{j=1}^{N_{C}} A_{i j} x_{i} x_{j}, A_{i j}=\sqrt{A_{i} A_{j}}\left(1-k_{i j}\right)$ and $B=\sum_{i=1}^{N_{C}} B_{i} x_{i} . \quad$ Therefore, at constant $\mathrm{T}$ and $\mathrm{P}$, the fugacity coefficient becomes a function of composition. Reduced methods aim to express the fugacity coefficient in fewer parameters than the number of components. Li and Johns (2006) and Okuno et. al. (2010a) express the 'A' term as $A=\Theta_{1}^{2}+2 \Theta_{2}^{2}-2 \Theta_{3} \Theta_{4}$ and hence the fugacity coefficient becomes a function of five $\quad\left[\Theta_{1}, \Theta_{2}, \Theta_{3}, \Theta_{4}, B\right]$ where $\Theta_{\alpha}=\left\{\sum_{i=1}^{N_{C}} x_{i} \sqrt{A_{i}}, \sum_{i=1}^{N_{C}} x_{i} \sqrt{A_{i}} h_{i} g_{i}, \sum_{i=1}^{N_{C}} x_{i} \sqrt{A_{i}} h_{i}^{2} g_{i}, \sum_{i=1}^{N_{C}} x_{i} \sqrt{A_{i}} g_{i}\right\}$. Nichita and Graciaa (2011), Nichita and Petitfrere (2013), and Gorucu and Johns (2014) linearize the natural logarithm of fugacity coefficient as

$$
\ln \phi_{i}=\sum_{\alpha=1}^{m} h_{\alpha} q_{i \alpha}
$$

where $m$ is the number of reduced variables, $h_{\alpha}$ are the reduced variables, and $q_{i \alpha}$ is a matrix of constant parameters. For the reduced variables of Gorucu and Johns (2014), $m$ is always 6 and the parameters in Eq. (4) are

$$
\begin{gathered}
h_{\alpha}=\frac{-2 \Theta_{\alpha}}{\Delta B} \ln \left(\frac{Z+\delta_{1} B}{Z+\delta_{2} B}\right) \quad \alpha=1, \ldots, 4 \\
h_{5}=\frac{(Z-1)}{B}+\frac{A}{\Delta B^{2}} \ln \left(\frac{Z+\delta_{1} B}{Z+\delta_{2} B}\right) \\
h_{6}=-\ln (Z-B) \\
q_{i \alpha}=\left\{\sqrt{A_{i}}, 2 \sqrt{A_{i}} h_{i} g_{i},-\sqrt{A_{i}} g_{i},-\sqrt{A_{i}} h_{i}^{2} g_{i}, B_{i}, 1\right\} .
\end{gathered}
$$

Our reduced algorithm based on these reduced parameters is referred to here as the Improved Li and Johns (ILJ) method since it uses the same two-parameter binary interaction formula. The parameters and approach are explained in detail in Gorucu (2013). 
The algorithm is similar to that of Nichita and Petitfrere (2013), although they used the reduced variables based on eigenvalue decomposition. The eigenvalue decomposition approach is slower owing to an undetermined number of parameters (Gorucu and Johns, 2015) and is therefore not likely to be used to speed up compositional simulation as explained by Haugen and Beckner (2013).

The reduced parameters given by Eqs. (5) - (7) are used in both stability and flash calculations. Our residual function for stability analysis is

$$
R_{\alpha}=h_{\alpha}^{n+1}-h_{\alpha}^{n}=0 \quad \text { for } \alpha=1, \ldots, 6
$$

where $n$ is the iteration number. The reduced variables are updated with

$$
\overline{\bar{J}} \Delta \bar{h}=-\bar{R}
$$

where $\Delta \bar{h}=\bar{h}^{n+1}-\bar{h}^{n}$. The Jacobian matrix is

$$
\boldsymbol{J}=\frac{\partial R_{\alpha}}{\partial h_{k}}=\frac{\partial h_{\alpha}^{n+1}}{\partial h_{k}^{n}}-\delta_{\alpha k}
$$

Once we solve for the independent variables $h_{\alpha}^{n+1}$, we calculate the fugacity coefficient using

$$
\ln \phi_{i}=\sum_{\alpha=1}^{6} h_{\alpha} q_{i \alpha}
$$

Subsequently, we update $X_{i}$ and $x_{i}$ with

$$
\begin{gathered}
X_{i}=\frac{z_{i} \phi_{i}(\bar{z})}{\phi_{i}(\bar{X})}, \\
x_{i}=\frac{X_{i}}{\sum_{j}^{N_{C}} X_{j}} .
\end{gathered}
$$

We switch from successive substitution to Newton-Raphson (NR) iterations when $\|S\|_{\infty}<\varepsilon$ where

$$
S_{i}=\ln X_{i}+\ln \phi_{i}(\vec{X})-\ln \left(z_{i} \phi_{i}(\vec{z})\right)=0
$$

and the value of the tolerance used $(\varepsilon)$ varies to test robustness. More robust methods can successfully converge using Newton iteration with a smaller $\varepsilon$. That is, more robust methods have a larger radius of convergence. Normally, $\varepsilon$ is set to $1 \times 10^{-3}$ or $1 \times 10^{-4}$ in compositional simulation, although for robust methods it may be possible to skip SS steps. 
The procedure for stability analysis using ILJ is summarized as follows, where we start with successive substitution (SS) iterations and switch to NR iterations. The procedure for SS iterations is,

1. Calculate A and B in the cubic equation-of-state. We used the Peng and Robinson EOS (PREOS) for all stability and flash calculations in this paper (Peng and Robinson, 1978).

2. Calculate the compressibility factor from PREOS, where the correct root is selected that is real, positive, and gives the smallest Gibbs free energy. While calculating the Gibbs free energy, the fugacity coefficient is stored so that it can be used in step 4).

3. Check whether the switch criterion is satisfied as shown in Eq. (15). If the criterion is met, switch to NR iterations. Otherwise continue with step 4).

4. Once the fugacity coefficient is updated with Eq. (12), Eqs. (13) and (14) are repeated to update the phase compositions. Go back to step 1).

The $h$ value used in Eq. (9) is stored at the last iteration of SS since it is also used in the NR iterations. The NR iterations begin after the SS iteration procedure is completed. The procedure for NR iterations is as follows:

1. Calculate A and B from the PREOS.

2. Calculate the compressibility factor, where again the correct compressibility factor must be selected. The $h$ value is stored. The dimensionless molar Gibbs energy for a particular phase can be calculated with $G=\sum_{i=1}^{N_{C}} x_{i} \ln f_{i}$.

3. Update the residuals using Eq. (9).

4. Construct the Jacobian matrix with Eq. (11).

5. Update the $h$ value with Eq. (10).

6. Update the fugacity coefficient with Eq. (12).

7. Repeat Eqs. (13) and (14) to update the composition.

8. Check for convergence with $\varepsilon=1 \times 10^{-10}$ in Eq. (15). If convergence is achieved, stop. Otherwise, go back to step 1).

This procedure avoids additional SS steps required by OJS as explained in Okuno et al. (2010b).

The derivatives for the Jacobian matrix construction are

$$
\frac{\partial h_{\alpha}^{n+1}}{\partial h_{k}^{n}}=\sum_{\gamma=1}^{5} \frac{\partial h_{\alpha}^{n+1}}{\partial \Theta_{\gamma}} \frac{\partial \Theta_{\gamma}}{\partial h_{k}^{n}} .
$$

The expressions for $\frac{\partial h_{\alpha}^{n+1}}{\partial \Theta_{\gamma}}$ are the same as in the two-phase flash calculations given in Gorucu and Johns (2014), while the analytical derivatives for $\frac{\partial \Theta_{\gamma}}{\partial h_{k}^{n}}$ are different for stability analysis than in flash calculations. The equations for the derivatives in stability analysis are found below.

$$
\frac{\partial Q_{\gamma j}}{\partial h_{\beta}}=\sum_{i=1}^{N_{c}} \theta_{i \gamma} \frac{\partial x_{i}}{d h_{\beta}}
$$

where $\theta_{i \gamma}=\left\{\sqrt{A_{i}}, \sqrt{A_{i}} h_{i} g_{i}, \sqrt{A_{i}} h_{i}^{2} g_{i}, \sqrt{A_{i}} g_{i}, B_{i}\right\}$. The required analytical derivatives in Eq. (17) are 


$$
\frac{\partial x_{i}}{\partial h_{\alpha}}=\left(Q_{\alpha}-q_{i \alpha}\right) x_{i}
$$

and,

$$
\frac{\partial X_{i}}{\partial h_{\alpha}}=-\frac{z_{i} \phi_{i}^{z}}{\phi_{i}^{\beta}} \frac{\partial \ln \phi_{i}^{\beta}}{\partial \partial h_{\alpha}}=-q_{i \alpha} X_{i} .
$$

\section{Three-Phase Flash Calculations Using New Reduced Variables}

We implemented the ILJ reduced variables to three-phase flash calculations that are described in detail in Gorucu (2013). Nichita and Petitfrere (2015) and Petitfrere and Nichita (2015b) recently showed that these reduced variables are unbounded and better scaled. These authors, however, used eigenvalue decomposition, while we used the empirical BIP formula of Li and Johns (2006). Like stability analysis, the equations are solved using SS iterations followed by NR iterations. For three-phase equilibrium calculations there are 12 independent variables, $6\left(N_{P^{-}}-1\right)$, required to determine and update the $K$-values.

The residual functions are given below:

$$
\begin{array}{ll}
e_{\alpha}=h_{\alpha}^{3}-h_{\alpha}^{1}-\Delta h_{\alpha}^{13} & \alpha=1, \ldots, 6 \\
e_{\alpha}=h_{\alpha}^{3}-h_{\alpha}^{2}-\Delta h_{\alpha}^{23} & \alpha=7, \ldots, 12
\end{array}
$$

The independent variables are,

$$
\psi_{k}=\left\{\Delta h_{1}^{13}, \Delta h_{2}^{13}, \Delta h_{3}^{13}, \Delta h_{4}^{13}, \Delta h_{5}^{13}, \Delta h_{6}^{13}, \Delta h_{1}^{23}, \Delta h_{2}^{23}, \Delta h_{3}^{23}, \Delta h_{4}^{23}, \Delta h_{5}^{23}, \Delta h_{6}^{23}\right\}
$$

where $\Delta h_{\alpha}^{13}=h_{\alpha}^{3}-h_{\alpha}^{1}$ and $\Delta h_{\alpha}^{23}=h_{\alpha}^{3}-h_{\alpha}^{2}$. Superscripts 1,2 and 3 are the phase labels. $K$-values are expressed as

$$
\ln K_{i 2}=\sum_{\alpha=1}^{6} \Delta h_{\alpha}^{23} q_{\alpha i} \quad \text { and } \quad \ln K_{i 1}=\sum_{\alpha=1}^{6} \Delta h_{\alpha}^{13} q_{\alpha i}
$$

The procedure starts with SS iterations based on initial $K$-values from converged stability analysis and subsequent two-phase flash calculations. NR iterations continue following SS iterations when the switch criterion is met. SS iterations are as follows:

1. Do constant-K flash calculations using the procedure of Okuno et al. (2010c).

2. Calculate fugacity coefficients with Eq. (12).

3. Check if the switch criterion is satisfied based on a similar equation as Eq. (15), but where $\left\|R_{i}\right\|_{\infty}=\left\|\ln \phi_{i}\left(\overline{x^{1}}\right)+\ln x_{i}^{1}-\ln \phi_{i}\left(\overline{x^{\beta}}\right)-\ln x_{i}^{\beta}\right\|_{\infty}<\varepsilon$. Here, $\beta$ are the second and third phases.

4. If the switch criterion is met, exit. Otherwise continue to step 1).

For the ILJ approach, one additional constant-K flash calculation is repeated between the SS and NR iterations in order to update the phase compositions. NR iterations are as follows:

1. Calculate fugacity coefficients with Eq. (12). 
2. Check for convergence using $\left\|R_{i}\right\|_{\infty}=\left\|\ln \phi_{i}\left(\overline{x^{1}}\right)+\ln x_{i}^{1}-\ln \phi_{i}\left(\overline{x^{\beta}}\right)-\ln x_{i}^{\beta}\right\|_{\infty}<10^{-10}$. If convergence is achieved, stop. Otherwise, continue with step 3).

3. Update the residuals with Eq. (20).

4. Construct the Jacobian matrix as shown in the Appendix.

5. Update independent variables by using $\overline{\Delta h}^{n+1}=\overline{\Delta h}^{n}-\overline{\mathbf{J}}^{-1} \bar{e}$. LU decomposition or Gaussian elimination can be used.

6. Update $K$-values with Eq. (22).

7. Do constant-K flash calculations as shown in Okuno et al. (2010c).

8. Go back to step 1 .

\section{Initialization, Switch and Convergence Criterion}

This section describes in more detail the methodology to initialize the $K$-values, switch from SS to NR iterations, and the convergence criterion for the three-phase equilibrium calculations. Phase equilibrium calculations begin with a single-phase stability analysis, followed by two-phase flash calculations if that result is unstable. Once two-phase flash calculations are complete, one of the two phases (preferably the one with the smallest Gibbs energy) is tested for stability in what we refer to here as a two-phase stability analysis. Three-phase flash calculations follow the two-phase stability analysis, but only if that phase is found to be unstable. Thus, the robustness of three-phase flash calculations are partially dependent on the robustness of the two-phase calculations.

\subsection{Stability analysis}

Single-phase stability analysis is initialized with the following $K$-values (Li \& Firoozabadi, 2012).

$$
K_{i}^{1 \phi-s t a b}=\left\{K_{i}^{\text {Wilson }}, 1 / K_{i}^{\text {Wilson }}, \sqrt[3]{K_{i}^{\text {Wilson }}}, 1 / \sqrt[3]{K_{i}^{\text {Wilson }}}, K_{i}^{\mathrm{CO}_{2}}\right\}
$$

where

$$
K_{i}^{C O_{2}}= \begin{cases}0.9 / z_{i}^{\text {test }} & \text { if } \mathrm{i}=\mathrm{n}_{\mathrm{CO}_{2}} \\ 0.1 /\left(N_{C}-1\right) / z_{i}^{\text {test }} & \text { if } \mathrm{i} \neq \mathrm{n}_{\mathrm{CO}_{2}}\end{cases}
$$

and $i$ is the component index, and $\mathrm{n}_{\mathrm{CO}_{2}}$ is the $\mathrm{CO}_{2}$ identifier. Two-phase stability analysis is initialized with (Li \& Firoozabadi, 2012).

$$
K_{i}^{2 \phi-s t a b}=\left\{K_{i}^{\text {Wilson }}, 1 / K_{i}^{\text {Wilson }}, \sqrt[3]{K_{i}^{\text {Wilson }}}, 1 / \sqrt[3]{K_{i}^{\text {Wilson }}}, K_{i}^{\text {CO }_{2}}, K_{i}^{1 \phi-\text { stab }}, 1 / K_{i}^{1 \phi-\text { stab }}, K_{i}^{2 \phi-\text { flash }}, 1 / K_{i}^{2 \phi-\text { flash }}\right\}
$$

Once the initial $K$-value estimates are computed, stationary points can be found by iterating with the variable 


$$
X_{i}=K_{i}^{\text {stab }} z_{i}^{\text {test }}
$$

where $z_{i}^{\text {test }}$ is the initial composition tested for stability. For single-phase stability analysis $z_{i}^{\text {test }}=z_{i}$. For two-phase stability analysis, $z_{i}^{\text {test }}$ is the phase composition using the equilibrium composition from the two-phase flash calculations with the lowest molar Gibbs energy. The molar residual Gibbs energy for a phase is calculated as

$$
G_{j}=\sum_{i=1}^{N_{C}} x_{i}^{j} \ln \left(\phi_{i}^{j} x_{i}^{j}\right)
$$

where $i$ is component index, $j$ is phase index, $x$ is the phase composition, and $\phi$ is the fugacity coefficient.

The convergence criterion used is

$$
\left\|S_{i}\right\|_{\infty}=\left\|\ln \phi_{i}(\bar{X})+\ln X_{i}-\ln \phi_{i}(\bar{z})-\ln z_{i}\right\|_{\infty}<10^{-10}
$$

At the end of every stability analysis, we first check whether convergence is achieved within a maximum number of iterations e.g. 20. We then also check whether a trivial solution is reached, and whether a positive TPD is achieved, or whether the stationary point is undefined (NaN). If none of these cases are achieved, the single phase being tested is unstable and subsequent flash calculations are made. The trivial solution for single-phase stability analysis is recognized when $\left\|X_{i} / z_{i}\right\|_{\infty}<10^{-8}$. Trivial solutions for two-phase stability analysis are identified when $\left\|X_{i} / x_{i}\right\|_{\infty}<10^{-8}$ or $\left\|X_{i} / y_{i}\right\|_{\infty}<10^{-8}$.

\subsection{Flash Calculations}

The initial $K_{i}$ values for two-phase flash calculations are

$$
K_{i}^{2 \phi-f l a s h}=\left\{K_{i}^{1 \phi-s t a b}, K_{i}^{\text {Wilson }}\right\}
$$

from Li \& Firoozabadi (2012). $K_{i}^{\text {Wilson }}$ values are initialized using Wilson (1969). Baker (1980) shows for binary mixtures that flash calculations do not guarantee convergence to the global minimum. Therefore, we carry out two-phase flash calculations several times even if the first estimate converges to a physical two-phase solution. We want to find the two-phase solution that gives the lowest Gibbs energy, where the residual Gibbs energy is calculated by

$$
G_{R}=\sum_{j=1}^{N_{P}} \sum_{i=1}^{N_{C}} \beta^{j} x_{i}^{j}\left(\ln \phi_{i}^{j} x_{i}^{j}\right)
$$

and $\beta$ is the phase mole fraction and $N_{P}$ is the number of phases. This strategy not only improves the results for two-phase flash calculations but also improves the initial estimates for two-phase stability analysis and subsequent three-phase flash calculations.

Three-phase flash calculations begin with the following two $K_{i}$ estimates namely 


$$
K_{i 1}=\frac{x_{i}}{X_{i}} \quad \text { and } \quad K_{i 2}=\frac{y_{i}}{X_{i}}
$$

where $x_{i}$ and $y_{i}$ are phase compositions from the two-phase flash calculations and $X_{i}$ is the stationary point from two-phase stability analysis. Two $K$-values are required as there are three phase compositions which are

$$
K_{i 1}=K_{i}^{2 \phi-s t a b} \quad \text { and } \quad K_{i 2}=K_{i}^{2 \phi-f l a s h}
$$

We use the constant-K flash calculation procedure outlined in Okuno et al. (2010c), since this procedure guarantees convergence for both two- and three-phase constant K-value calculations. The convergence criterion for two-phase flash calculations is

$$
\left\|R_{i}\right\|_{\infty}=\left\|\ln \phi_{i}(\bar{x})+\ln x_{i}-\ln \phi_{i}(\bar{y})-\ln y_{i}\right\|_{\infty}<10^{-10}
$$

We use two convergence criteria for three-phase flash calculations:

$$
\left\|R_{i}\right\|_{\infty}=\left\|\ln \phi_{i}\left(\overline{x^{1}}\right)+\ln x_{i}^{1}-\ln \phi_{i}\left(\overline{x^{2}}\right)-\ln x_{i}^{2}\right\|_{\infty}<10^{-10}
$$

and

$$
\left\|R_{i}\right\|_{\infty}=\left\|\ln \phi_{i}\left(\overline{x^{1}}\right)+\ln x_{i}^{1}-\ln \phi_{i}\left(\overline{x^{3}}\right)-\ln x_{i}^{3}\right\|_{\infty}<10^{-10} .
$$

If the first estimate successfully converges to a non-trivial physical solution, the second initial guess is not carried out. At the end of each flash calculation, we first check whether the maximum number of iterations is reached, then if a non-physical solution is achieved, and last if the solution is undefined $(\mathrm{NaN})$, or a trivial solution is achieved. Undefined solutions can occur when one of the phase compositions become negative. If none of these conditions are satisfied, the flash calculation is assumed to converge to a non-trivial physical solution. A trivial solution for two-phase flash calculations is assumed when $\sqrt{\sum_{i=1}^{N_{C}}\left(x_{i}-y_{i}\right)^{2}}<10^{-5}$ and $\sqrt{\sum_{i=1}^{N_{C}}\left(z_{i}-y_{i}\right)^{2}}<10^{-5}$. For three-phase flash calculations, the trivial solution is reached when either $\left\|K K_{i 1}-1\right\|_{\infty}<10^{-5}$ or $\left\|K K_{i 2}-1\right\|_{\infty}<10^{-5}$ conditions are satisfied.

\subsection{Strategy for Testing and Comparison}

We tried different strategies for testing ILJ, OJS and the minimization of Gibbs energy technique (MG). The strategy relied on determining a "truth model" based on performing SS iterations alone until a very small tolerance was achieved. This truth model was then used to identify when the conventional and reduced methods that use Newton iterations do not converge. The strategy is summarized by, ,

1) Make phase equilibrium calculations with SS iterations using a $10^{-10}$ tolerance in the convergence

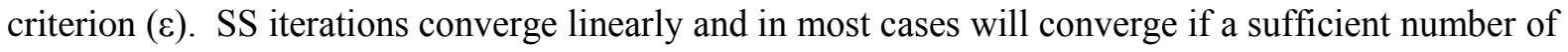
iterations are allowed as long as the overall composition does not lie too close to a critical point. Because convergence is very slow near critical points, we allowed for a maximum number of SS 
iterations of one million. The solution obtained from these SS iterations is taken to be the correct solution because it is most likely to converge compared to Newton iterations, albeit in a very long computational time.

2) For the conventional and reduced methods, we start with SS and switch to IL-NR (or OJS-NR) by varying values of the switch criterion from $10^{-1}, 10^{-2}$, to $10^{-3}$. The maximum number of SS and NR iterations are 100 and 20 , respectively.

3) We also made a test of the conventional and reduced methods where only one SS iteration was used independent of the convergence criterion. For such cases, the maximum number of NR iterations are 20 for both ILJ-NR and OJS-NR.

4) Convergence criterion for the NR iterations is always based on the fugacity equations and is set to $10^{-}$

${ }^{10}$ for all conventional and reduced methods.

\section{Results and Discussion}

In this section we compare two reduced methods (ILJ and OJS) with the conventional method ( MG) for their ability to converge with three-phase equilibrium calculations. The test data used in the comparison are pressure-temperature (PT), and pressure-composition (PX) diagrams for two fluids that are well characterized in the literature. These fluids are the North Ward Estes (NWE) fluid from Khan et al. (1992), and the Maljamar separator oil from Orr et al. (1981). The $h_{i}$ and $g_{i}$ vectors (BIP parameters) for the NWE fluid and Maljamar separator oil can be found in table 1.

Phase equilibrium calculations are more robust when only SS iterations are carried out. However, SS iterations are very slow. Therefore, we switch to NR iterations after several SS iterations and compare the results with the reference case, i.e. using SS iterations only. The use of a small convergence criterion for the SS iterations may cause less robust methods to fail. That is, the method with the largest radius of convergence will fail more often as the SS switch criterion is made smaller.

We discretized the PT diagram of the NWE fluid into 30,844 equally-spaced points and made three-phase equilibrium calculations at each point using ILJ, OJS, and MG. In table 2, we report the results for the NWE fluid. The table contains four main sections in the sequence normally taken, namely single-phase stability analysis, two-phase flash calculations, two-phase stability analysis, and three-phase flash calculations. For stability analysis of a single-phase mixture, we report the average number of SS iterations, average number of NR iterations, how many $K$-value initializations on average were required to achieve a negative tangent plane distance (TPD) indicating an unstable phase, and what percentage of the calculations detected a second phase. The reported SS and NR iterations belong to the final stability analysis only. That is, only SS and NR iterations from the stability analysis that give two-phase solutions are reported. Almost all of the stability analysis calculations converged to negative TPD at the first Kvalue initialization. Only a few required a second initial $K_{i}$ estimation. The number of SS iterations are identical and NR iterations varied slightly between ILJ and OJS. MG converged in significantly more Newton iterations than ILJ and OJS. 100\% convergence, which is shown in table 2, means that all of the stability analyses successfully identified a second phase.

Table 2 also reports the results for the NWE fluid for two-phase flash calculations. For twophase flash calculations, both initializations are carried out. However, only the solution with lower Gibbs energy is reported. Therefore, the reported SS and NR iterations belong to the corresponding flash calculation only. When SS $\left(10^{-10}\right)$ convergence criterion is used, nearly all of the flash calculations converged to a non-trivial two-phase solution. The results from each SS iteration are exactly the same prior to performing ILJ, OJS and MG. ILJ converges in fewer number of NR iterations than MG and OJS. The slight difference is caused by the fact that initializations are not always the same because stability analysis did not always did not always give the same conclusion. When SS switches to NR at $10^{-}$ 
${ }^{3}$ convergence criterion, ILJ, OJS and MG converge to a non-trivial two-phase solution for almost all of the cases. When the SS switch criterion is relaxed to 0.1, OJS and MG fail more often than ILJ. That is, ILJ, OJS, and MG converged to a two-phase solution in $98.3 \%, 94.7 \%$, and $94.3 \%$ of the cases. This indicates that ILJ is somewhat more robust than OJS and MG for these two-phase flash calculations. The improved robustness in the two-phase region will also give better robustness for finding the three-phase region since the three-phase flash calculations depend on the prior two-phase results.

The two-phase stability analysis section of table 2 reports the average number of SS, NR iterations, initializations and what percentage of the two-phase stability analyses were able to identify a possible third phase, as compared to those previously identified when SS $\left(10^{-10}\right)$ convergence criterion was used. The average number of initializations is reported only if the stability analysis identified a third phase. Two-phase stability analysis always gives a three-phase solution using only the first three initializations from Eq. (25). That is, the other initializations were never needed. The reported SS and NR iterations in Table 2 correspond to the last stability analysis iteration only. The average number of initializations and NR iterations is similar for ILJ, OJS and MG. However, ILJ is able to identify the third phase more successfully than OJS and MG because OJS and MG failed more often in the two-phase flash calculation step, as described previously. Therefore, OJS and MG could not continue with the two-phase stability analysis step for these cases.

In table 2, the section on three-phase flash calculations reports the average number of SS, NR iterations, K-value initializations only for convergence to a non-trivial solution in the three-phase region, and the percentage of the three-phase region that MG, ILJ and OJS were able to successfully identify. ILJ requires more initializations at a smaller (relaxed) switch criterion. This is again due to the fact that ILJ was able to carry out three-phase flash calculations where OJS and MG failed for two-phase flash calculations. Moreover, ILJ converged in somewhat fewer NR iterations. Finally, ILJ, OJS and MG are able to identify $86.7 \%, 80 \%$ and $69.6 \%$ of the three-phase region calculations, respectively.

Figure 1 shows the results graphically. That is, Fig. 1 gives the number of phases converged to for the NWE oil calculated by SS iterations only, while figures 2-4 show the number of converged phases when SS switches to MG, OJS and ILJ using a more relaxed switch criterion of 0.1. The phases are identified successfully by using SS iterations and the results agree exactly with those in Okuno (2009). No single-phase region exists because of the high concentration of $\mathrm{CO}_{2}$. When MG, ILJ and OJS are used with a $10^{-3} \mathrm{SS}$ switch criterion, all results are robust as shown in table 2. However, when we relax the switch criterion to 0.1, ILJ, OJS and MG start to fail. Figures 2-4 show the results for two-phase flash calculations using the 0.1 switch criterion for MG, OJS and ILJ, respectively. The single-phase region appears here only because some of the two-phase flash calculations failed. Therefore, these results indicate that ILJ is significantly more robust in making two-phase flash calculations particularly in the three-phase region. That is, two-phase flash calculations are required to be computed before the threephase flash calculations. As OJS and MG failed in two-phase flash calculations more often, they incorrectly identified three-phase points as only one phase. Further, ILJ, OJS and MG converged to false two-phase points within the three-phase region, while ILJ was able to identify the third phase more successfully.

We further relaxed the switch criterion and switched to NR iterations only after one SS iteration, independent of the convergence criterion. The maximum number of NR iterations allowed was set to 20 . Figures 5-7 show the number of converged phases for MG, OJS and ILJ. The results show that ILJ phase equilibrium calculations are again significantly more robust than OJS and MG. MG did not converge for most compositions in the three-phase region, and incorrectly exhibited many single-phase points at high pressures, similarly to OJS.

Because two-phase flash calculations for MG and OJS failed to converge for many of the cases in the three-phase region, it is difficult to compare the two-phase stability analysis and three-phase flash calculation results from OJS, MG and ILJ. Therefore, we modified the strategy and carried out one-phase stability analysis, followed by two-phase flash calculations using only SS iterations until convergence was achieved with $\varepsilon=10^{-10}$. Then, we made OJS, MG and ILJ two-phase stability analysis using a SS switch criterion of $10^{-1}$. Using this strategy, OJS, MG and ILJ failed to identify the three-phase region 23, 
23, and 20 times out of 6317, respectively, when compared with the reference algorithm based on only SS iterations. Further, it took on average 2.13, 2.19 and 2.13 initializations for two-phase stability analysis with OJS, MG and ILJ in order to identify the third phase. Therefore, two-phase stability analysis using OJS, MG and ILJ analyses showed similar behavior in terms of robustness.

Next, we carried out conventional single-phase stability analysis, two-phase flash calculations, and two-phase stability analysis using SS iterations only with $\varepsilon=10^{-10}$ so that convergence was nearly always obtained. We then made one SS iteration for three-phase flash calculations and finally switched to Newton iterations using the MG, OJS and ILJ methods. Based on this strategy we could compare the three-phase flash calculation results using MG, ILJ and OJS with the same initialization. Figures 8-10 show the phase identification results for MG, OJS and ILJ respectively. MG, OJS and ILJ failed to identify the three-phase region 2547, 1584 and 1580 out of 6317, respectively, when compared with the reference algorithm based on pure SS iterations. These results show that ILJ and OJS three-phase flash calculations are more robust than MG in these three-phase flash calculations.

Figure 11 compares the convergence behavior for ILJ, OJS and MG stability analyses where the convergence behavior is based on Eq. (28) at 1000 psi and $50^{\circ} \mathrm{F}$. Figure 12 shows the number of NR iterations required for convergence for ILJ, OJS and MG stability analyses at 1000 psi and at varying temperature $\left(50-93{ }^{\circ} \mathrm{F}\right)$. All of the methods are initialized with the same $K$-values. The SS switch criterion used is $10^{-1}$, while the NR convergence criterion is $10^{-10}$. Figures 11 and 12 show that all of the compared methods converge in a similar number of iterations for stability analyses and were also nearly identical to the results in Gorucu and Johns $(2014,2015)$. However, ILJ shows faster convergence than the algorithms of Li and Johns (2006) and Hendriks and Van Bergen (1992) as explained by Gorucu and Johns $(2014,2015)$. Figure 13 also shows the convergence behavior of ILJ, OJS and MG for a threephase flash calculation at $66^{\circ} \mathrm{F}$ and 1000 psi when started with the same $K$-values. Figure 14 shows the number of iterations for ILJ, OJS and MG three-phase flash calculations between 66 and $79{ }^{\circ} \mathrm{F}$ at 1000 psi. The results all demonstrate that ILJ has a slightly faster convergence behavior (less required iterations) than MG and OJS. Similar results can be found in table 2. However, the difference is not significant.

ILJ two-phase and three-phase flash calculations are more robust than OJS and MG owing to their potential to achieve a negative phase mole fraction more often than ILJ as iterations proceed. This indicates that ILJ has a larger radius of convergence. Figure 15 shows the convergence behavior for ILJ, OJS and MG two-phase flash calculations at $800 \mathrm{psi}$, and $57^{\circ} \mathrm{F}$ for NWE oil, where all flash calculations began with $K$-values from single-phase stability analysis where initially only one SS iteration was done. ILJ eventually converged even though its residual function initially increased. Similarly, OJS converged after 18 iterations and MG diverged soon after one phase became negative. Figure 16 shows an example of this in that even though OJS converged to a solution, the solution gave a negative phase mole fraction. OJS phase mole fraction converged to -243406 , although for clarity all negative values were cutoff at -1 . For the same case, ILJ started with 0.567 liquid mole fraction but quickly converged to 0.232 . In Figure 17 , at 823 psi and $57^{\circ} \mathrm{F}, \mathrm{MG}$ and OJS converged to a negative phase mole fraction and eventually diverged, whereas ILJ converged to a nontrivial physical solution.

Even though ILJ is more robust than the other tested algorithms, it failed for several T and $\mathrm{P}$ values when started with a poor K-value initialization. We compared ILJ, OJS and MG two-phase flash calculations using K-values from only the stability analysis and Wilson's correlation. NWE oil at $50{ }^{\circ} \mathrm{F}$ and 902 psi is chosen for comparison. This PT data selected is in a three-phase region but very close to the two-phase region. The Newton iterations begin after one SS iteration to test robustness. The initial Kvalues are given in table 3. When started with Wilson's K-values, all flash calculations diverged to a negative phase mole fraction. MG not only went to a negative phase mole fraction, but also converged to a negative phase composition after the first Newton iteration. Both OJS and ILJ diverged to -0.00174 vapor mole fraction after a few NR iterations. K-value initialization from the stability analysis gave a value of 0.09239 for the vapor phase mole fraction for ILJ and OJS. However, MG continued towards a more negative composition and eventually the iterations were stopped. Even though the correct solution is in the three-phase region (according to the truth model), the algorithm must successfully complete the 
two-phase flash calculations in order to continue on. We have found from the examples tested that the main advantage of ILJ is that its two-phase flash calculations are robust in the three-phase region.

Last, we made calculations with a different oil, the Maljamar separator oil (Orr et al. 1981), which has reported PX experimental data. We made a pressure- $\mathrm{CO}_{2}$ composition (PX) diagram for this crude by discretizing the diagram into $28336(176 \times 161)$ points. Figures $18-21$ show the results of phase identification using

1. SS iterations only with $\varepsilon=10^{-10}$ (similar strategy to give the truth model as for the NEW fluid).

2. One SS iteration with a maximum of 20 MG Newton iterations for all three methods (MG, OJS, and ILJ).

A $10^{-10}$ fugacity based convergence criterion is applied for all the algorithms for final convergence. Figure 18 gives the truth model from the SS iterations. The results in the figure match the reported data in Orr et. al. (1981) nearly exactly. The MG, OJS, and ILJ were successful in identifying the three-phase points $18.9,28.5$ and $44.9 \%$ of the time, respectively. Further, the two-phase region was correctly identified $43.7,60.3$ and $79.5 \%$ of the data points, as compared to the truth model. These results are consistent with the conclusions from the tests from the NWE fluid. That is, the ILJ method for multiphase equilibrium calculations are significantly more robust than OJS and MG. This improved robustness has the potential to facilitate convergence for three-phase compositional simulation.

\section{Summary and Conclusions}

We examined the robustness of three-phase flash calculations and stability analysis based on the empirical BIP formula of Li and Johns (2006) and the reduced variables of Gorucu (2013), Gorucu and Johns (2014) and Nichita and Petitfrere (2013). This method (ILJ) was compared to the three-phase equilibrium calculations using the reduced method of Okuno et al. (2010a) (OJS) and the conventional phase equilibrium calculations based on the minimization of Gibbs free energy (MG) (Perschke et. al. 1989, Michelsen and Mollerup 2007). Two fluids were used in the comparisons: the PT diagram of NWE oil from Khan et al. (1992) and a PX diagram of Maljamar separator oil from Orr et al. (1981). Our results suggest that

- ILJ, OJS and MG stability analysis have similar robustness. Thus, there is no clear advantage in using ILJ reduced methods or any other reduced method for stability analysis.

- ILJ two-phase flash calculations are more robust than OJS and MG two-phase flash calculations as was previously shown by Gorucu and Johns (2015).

- The robustness of two-phase flash calculations using ILJ in the three-phase region is significantly better than the other tested methods. That is, two-phase flash calculations with OJS and MG failed more often in the known three-phase region so that subsequent three-phase flash calculations could not be made. For these cases, OJS and MG misidentified three-phase points as a single phase.

- ILJ and OJS three-phase flash calculations are more robust than MG when started with the same $K$-values. For example, MG, OJS and ILJ failed to identify the three-phase region 2547, 1584 and 1580 times out of 6317 for NWE oil using one testing methodology (large SS switch criterion to Newton iterations).

- Generally, ILJ is more robust because OJS and MG tended to converge to the trivial solution more often for the cases studied. This indicates a larger radius of convergence for ILJ than the 
other methods. The larger radius of convergence could be related to the linearization of the fugacity coefficient expression.

Robust phase equilibrium calculations are very important when the initial $K$-values are far from the solution, as could exist in compositional simulation. For example, when implicit reservoir simulators are used, large time steps are desirable and initial $K$-values from a prior solution may be far from the next converged solution. Thus, a more robust algorithm for phase equilibrium calculations, such as that described here, could improve the convergence in compositional reservoir simulators, avoiding failure of the simulations.

Table 1. The $h_{\mathrm{i}}$ and $g_{i}$ parameters of Maljamar Separator oil and North Ward Estes fluid.

\begin{tabular}{|cccc|}
\hline \multicolumn{2}{|c}{ Maljamar Separator Oil } & \multicolumn{2}{c|}{ North Ward Estes Fluid } \\
\hline$h_{i}$ & $g_{i}$ & $h_{i}$ & $g_{i}$ \\
\hline 0 & 1 & 1 & 1 \\
0.339116 & 1 & 0 & 0.12 \\
0.339116 & 1 & 0 & 0.12 \\
0.339116 & 1 & 0 & 0.12 \\
0.339116 & 1 & 0 & 0.09 \\
0.339116 & 1 & 0 & 0.09 \\
0.339116 & 1 & 0 & 0.09 \\
\hline
\end{tabular}


Table 2. Summary of phase equilibrium calculations for NWE oil. SS: Average number of SS iterations. NR: Average number of NR iterations. Trial: Number of $K$-value initializations.

\begin{tabular}{|c|c|c|c|c|c|c|c|c|}
\hline & \multicolumn{4}{|c|}{ One-Phase Stability Analysis } & \multicolumn{4}{|c|}{ Two-Phase Flash Calculations } \\
\hline & SS & NR & Trial & Convergence $(\%)$ & SS & NR & & nvergence $(\%)$ \\
\hline SS (1E-10) & 21.63 & - & 1.02 & - & 47.33 & - & & - \\
\hline SS (1E-3)+OJS & 8.55 & 1.3 & 1.02 & 100 & 16.95 & 2.08 & & 99.98 \\
\hline SS (1E-3)+ILJ & 8.55 & 1.39 & 1.02 & 100 & 16.89 & 2.03 & & 99.96 \\
\hline $\mathrm{SS}(1 \mathrm{E}-3)+\mathrm{MG}$ & 8.55 & 1.72 & 1.02 & 100 & 16.64 & 2.04 & & 99.96 \\
\hline SS (1E-1)+OJS & 4.81 & 2 & 1.02 & 100 & 6.96 & 3.84 & & 94.7 \\
\hline SS (1E-1)+ILJ & 4.81 & 2.26 & 1.02 & 100 & 6.87 & 3.32 & & 98.3 \\
\hline \multirow[t]{3}{*}{ SS (1E-1)+MG } & 4.81 & 2.38 & 1.02 & 100 & 6.95 & 3.74 & & 94.3 \\
\hline & \multicolumn{4}{|c|}{ Two-Phase Stability Analysis } & \multicolumn{4}{|c|}{ Three-Phase Flash Calculations } \\
\hline & SS & NR & Trial & Convergence $(\%)$ & SS & NR & Trial & Convergence $(\%)$ \\
\hline SS (1E-10) & 100.07 & - & 2.12 & - & 104.33 & - & 1 & - \\
\hline SS (1E-3)+OJS & 19.39 & 1.93 & 2.12 & 99.78 & 22.74 & 2.23 & 1 & 98.88 \\
\hline SS (1E-3)+ILJ & 19.38 & 2 & 2.12 & 99.76 & 23.39 & 2.12 & 1 & 96.42 \\
\hline SS (1E-3)+MG & 19.41 & 2.12 & 2.13 & 99.75 & 23.75 & 2.1 & 1.02 & 96.06 \\
\hline SS (1E-1)+OJS & 5.45 & 2.88 & 2.14 & 84.14 & 3.43 & 4.15 & 1.01 & 80 \\
\hline SS (1E-1)+ILJ & 5.6 & 3.38 & 2.24 & 93.75 & 3.89 & 3.85 & 1.11 & 86.7 \\
\hline $\mathrm{SS}(1 \mathrm{E}-1)+\mathrm{MG}$ & 5.64 & 3.00 & 2.19 & 84.53 & 3.88 & 4.1 & 1.06 & 69.6 \\
\hline
\end{tabular}

Table 3. K-value initialization for two-phase flash calculations for NWE oil at $50{ }^{\circ} \mathrm{F}$ and 902 psi.

\begin{tabular}{|c|c|}
\hline Wilson & Stability analysis \\
\hline 0.727338 & 0.471279 \\
4.33945 & 0.578795 \\
0.198138 & 0.996926 \\
$7.14 \mathrm{e}-03$ & 1.34511 \\
$2.37 \mathrm{e}-05$ & 2.097708 \\
$2.51 \mathrm{e}-08$ & 5.445325 \\
$4.93 \mathrm{e}-13$ & 284.6891 \\
\hline
\end{tabular}




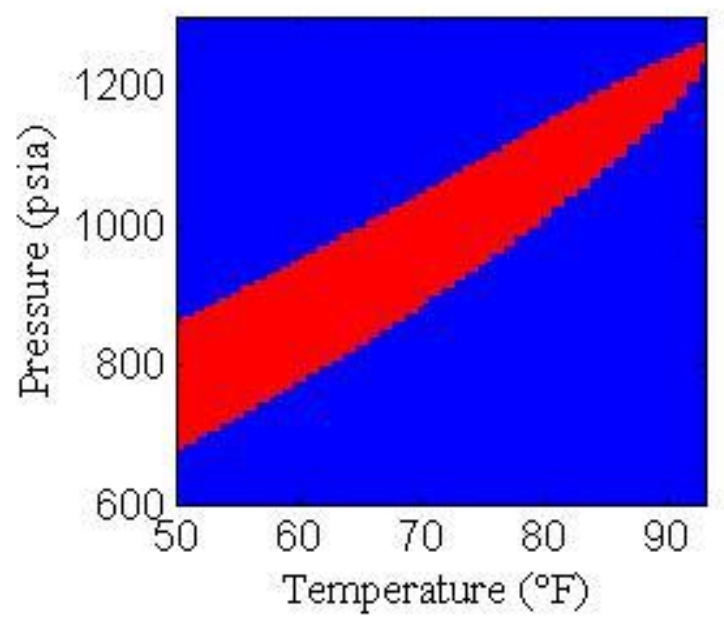

Figure 1. Identified number of phases for NWE oil resulting from phase equilibrium calculations using only SS iterations. Maximum number of SS iterations are one million. The blue region converged to the two-phase solution, while red points converged to three-phase points. This is the "truth model" for subsequent comparisons.

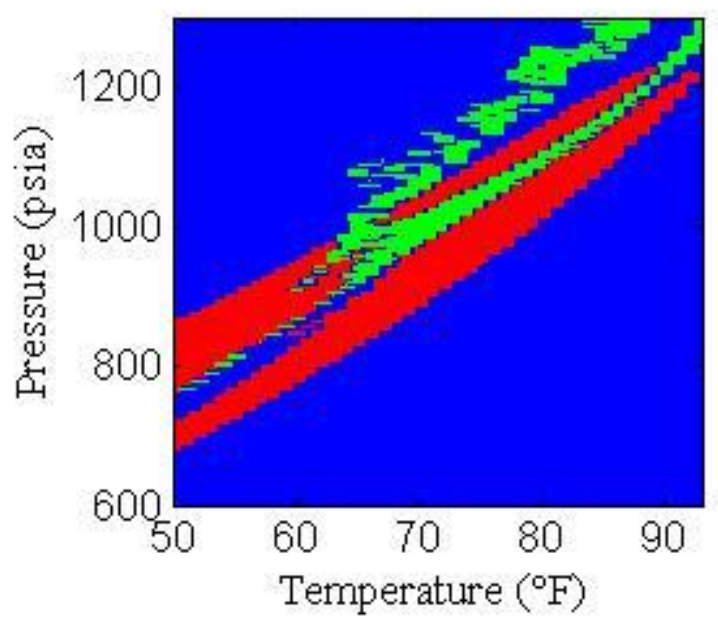

Figure 2. Identified number of phases for NWE oil when SS iterations switch to MG at a convergence criterion of $10^{-1}$. Green, blue and red regions converged to single-, two- and three-phase regions, respectively.

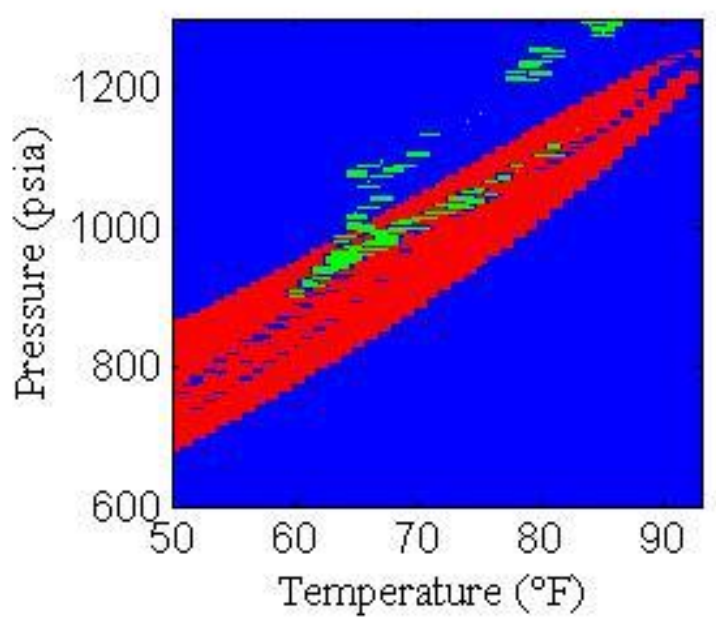

Figure 4. Identified phases for NWE oil when SS iterations switch to ILJ at $10^{-1}$ convergence criterion. Green, blue and red regions converged to single-, two- and three-phase regions, respectively. Convergence is improved compared to Fig. 3 that uses OJS. 


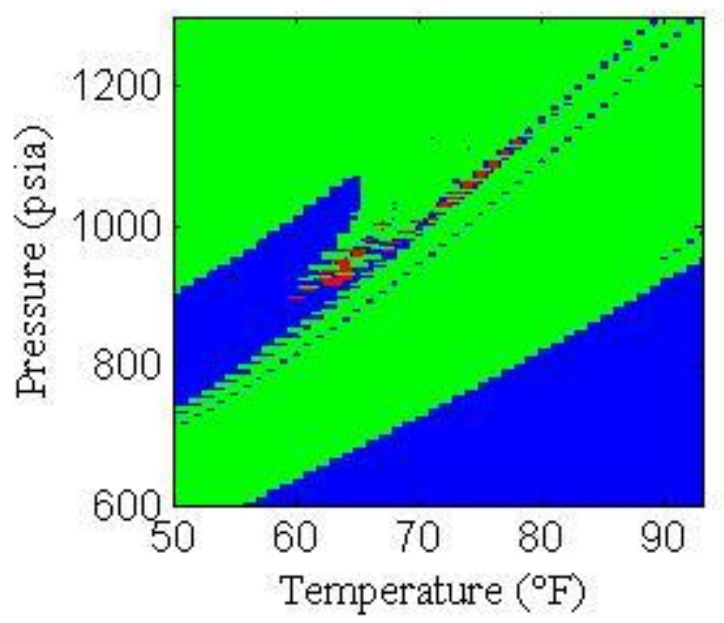

Figure 5. Identified phases for NWE oil when MG starts after only one SS iteration. Green, blue and red regions converged to single-, two- and three-phase regions, respectively. Convergence is very poor.

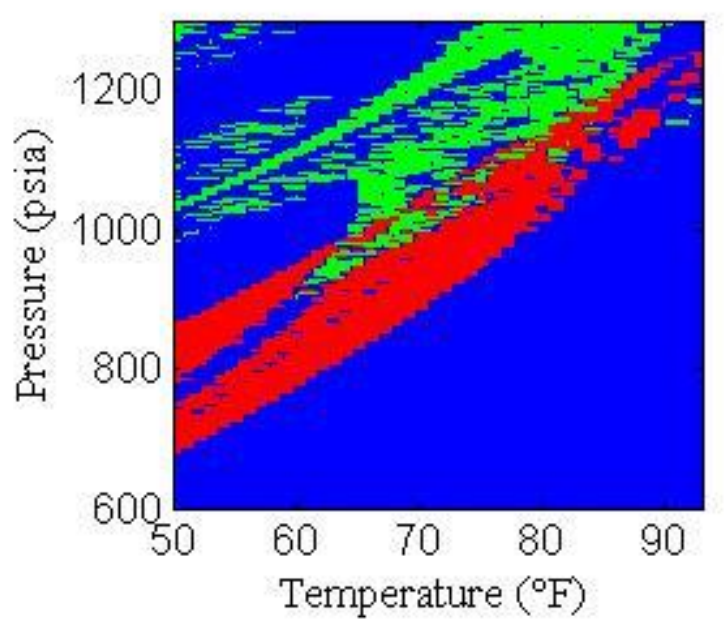

Figure 7. Identified phases for NWE oil when ILJ starts after only one SS iteration. Green, blue and red regions converged to single-, two- and three-phase regions, respectively. ILJ shows the best convergence behavior.

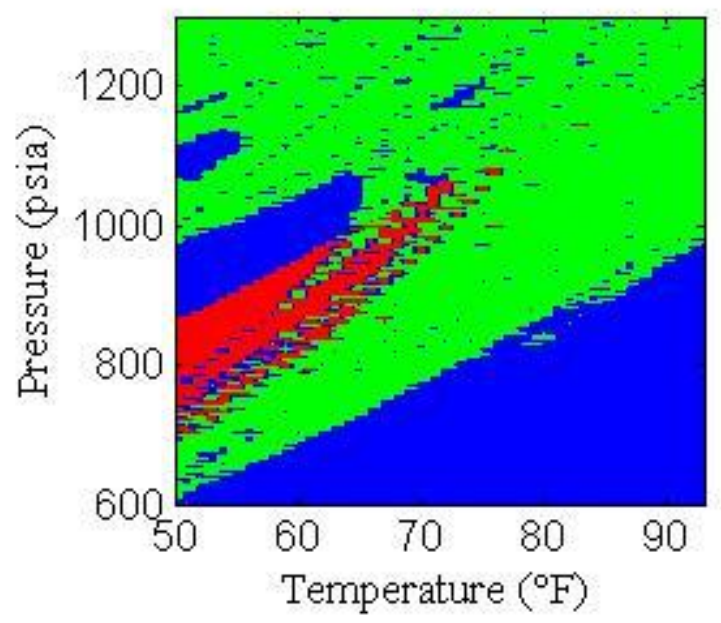

Figure 6. Identified phases for NWE oil when OJS starts after only one SS iteration. Green, blue and red regions converged to single-, two- and three-phase regions, respectively. Convergence is better than MG shown in Fig. 5.

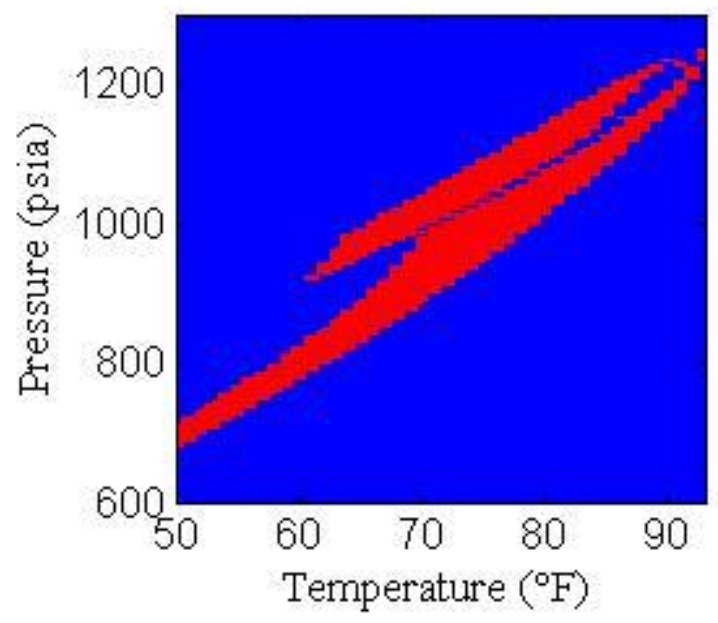

Figure 8. Number of phases for NWE oil. Conventional SS is carried out for single and two-phase flash calculations. MG starts after the first iteration of three-phase flash calculations. The blue region converged to two-phase solutions, while red points converged to three-phase points. 


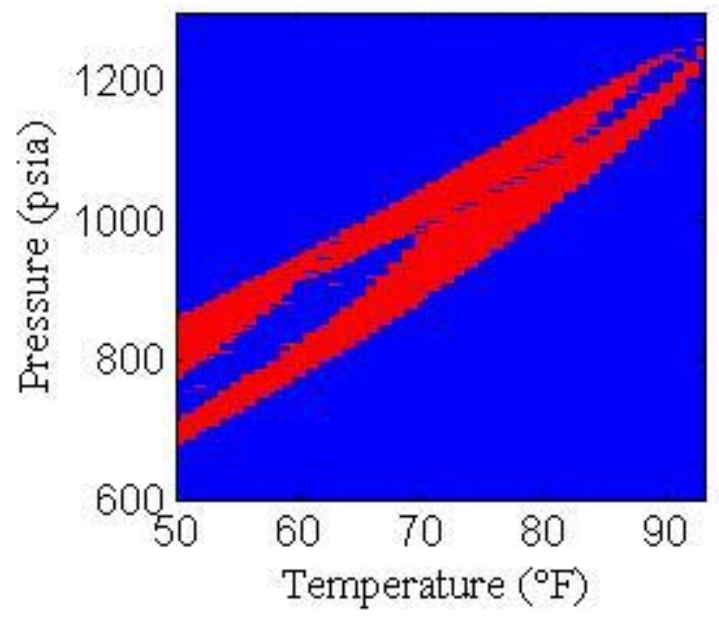

Figure 9. Number of phases for NWE oil. Conventional SS is carried out for single and two-phase flash calculations. OJS starts after the first iteration of three-phase flash calculations. The blue region converged to two-phase solutions, while red points converged to three-phase points.

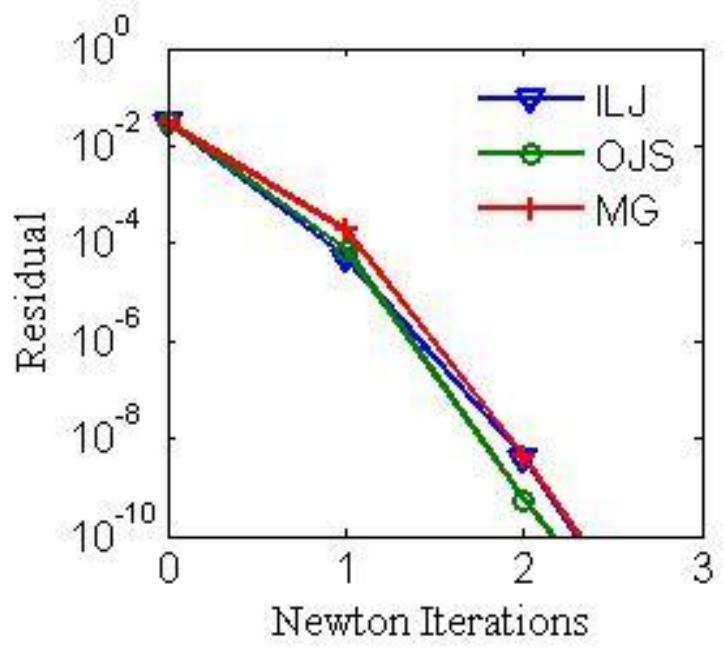

Figure 11. Convergence behavior for ILJ, OJS and MG stability analyses at $1000 \mathrm{psi}$ and $50^{\circ} \mathrm{F}$ for NWE oil. Similar convergence behavior is observed when fugacity based convergence criterion is used.

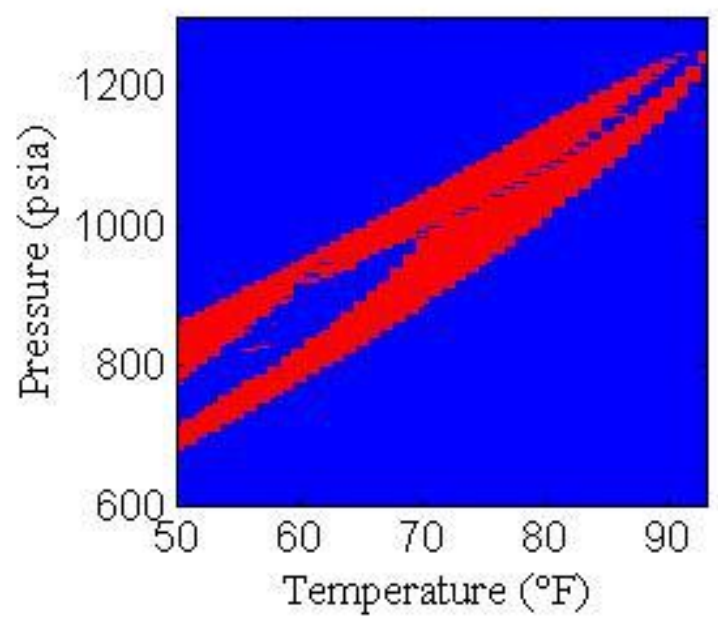

Figure 10. Number of phases for NWE oil. Conventional SS is carried out for single and two-phase flash calculations. ILJ starts after the first iteration of three-phase flash calculations. The blue region converged to two-phase solutions, while red points converged to three-phase points.

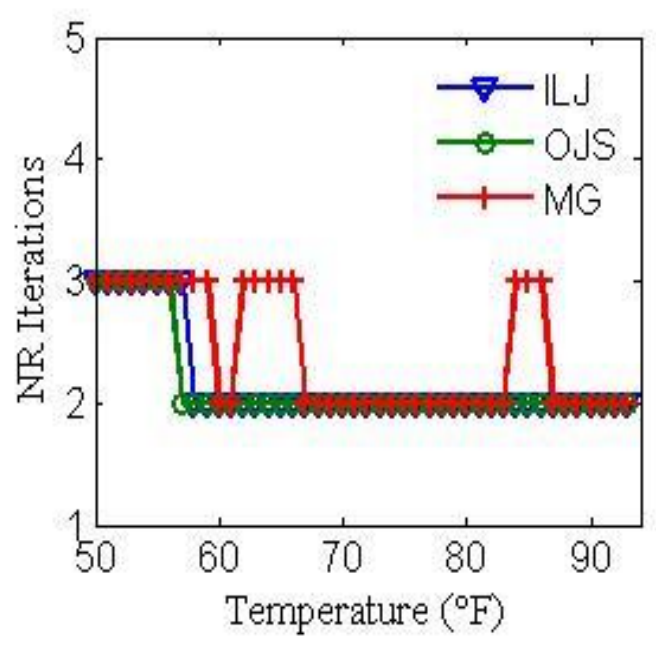

Figure 12. Newton iterations for ILJ, OJS and MG stability analyses for NWE oil at 1000 psi. Similar convergence behavior is observed when fugacity based convergence criterion is used. 


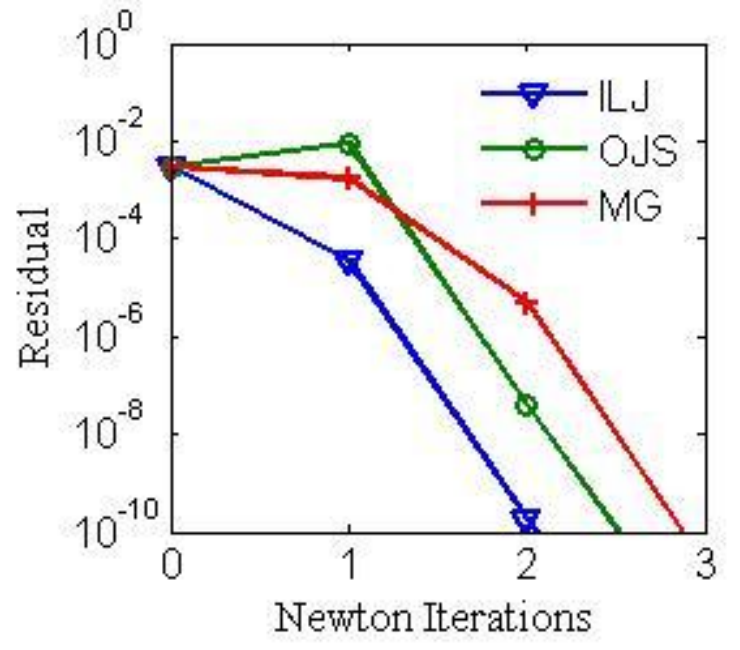

Figure 13. Convergence behavior for ILJ, OJS and MG three-phase flash calculations at $1000 \mathrm{psi}$ and $66^{\circ} \mathrm{F}$ for NWE oil. ILJ shows slightly improved convergence behavior.

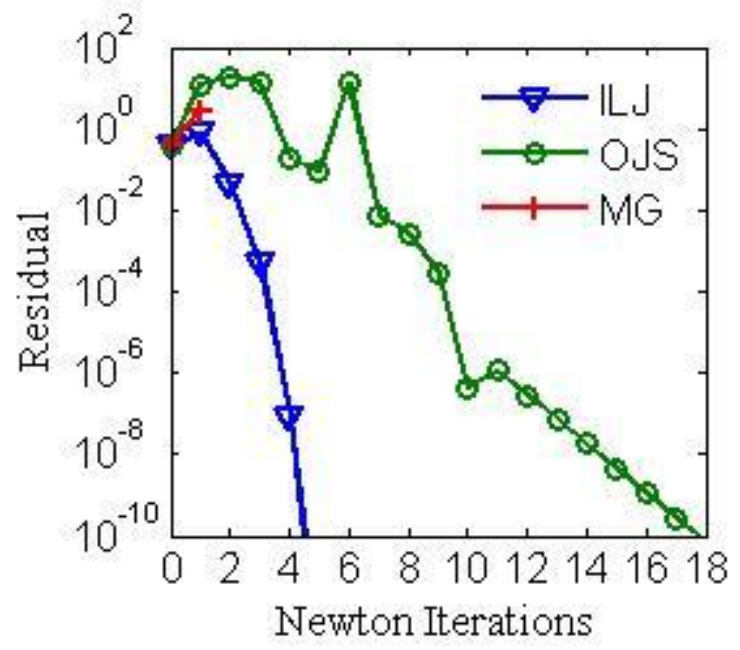

Figure 15. Convergence behavior for ILJ, OJS and MG two-phase flash calculations at 800 psi and $57^{\circ} \mathrm{F}$ for NWE oil. They all began with the same $K$-value initialization from the stability analysis with only one SS iteration.

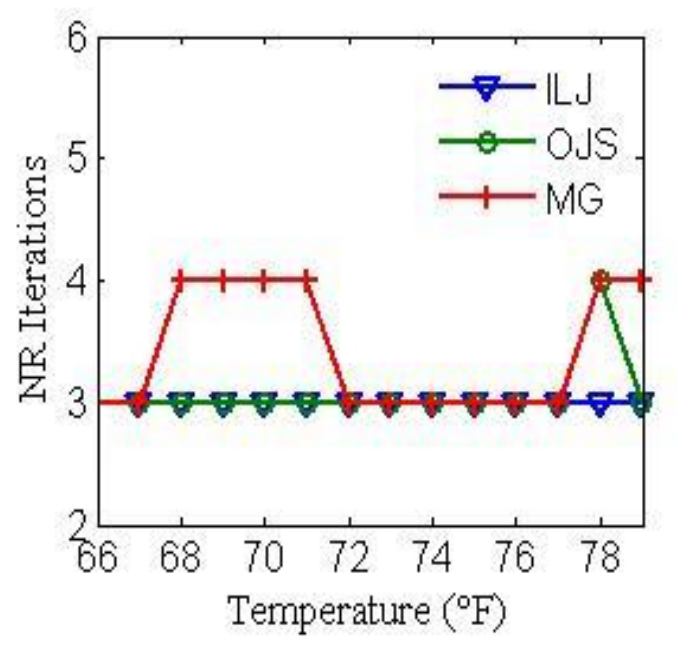

Figure 14. Newton iterations for ILJ, OJS and MG three-phase flash calculations for NWE oil at 1000 psi. ILJ always converges in three iterations while OJS and MG converge in three or four iterations.

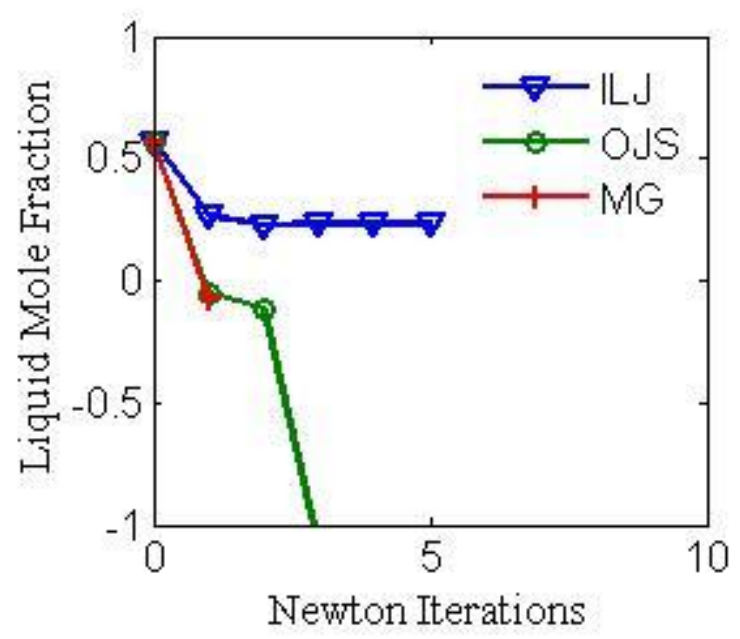

Figure 16. Liquid phase mole fraction for ILJ, OJS and MG two-phase flash calculations at $800 \mathrm{psi}$ and $57^{\circ} \mathrm{F}$ for NWE oil. ILJ converges to a nontrivial value that is between zero and one. MG and OJS quickly approached a negative phase mole fraction and diverged. 


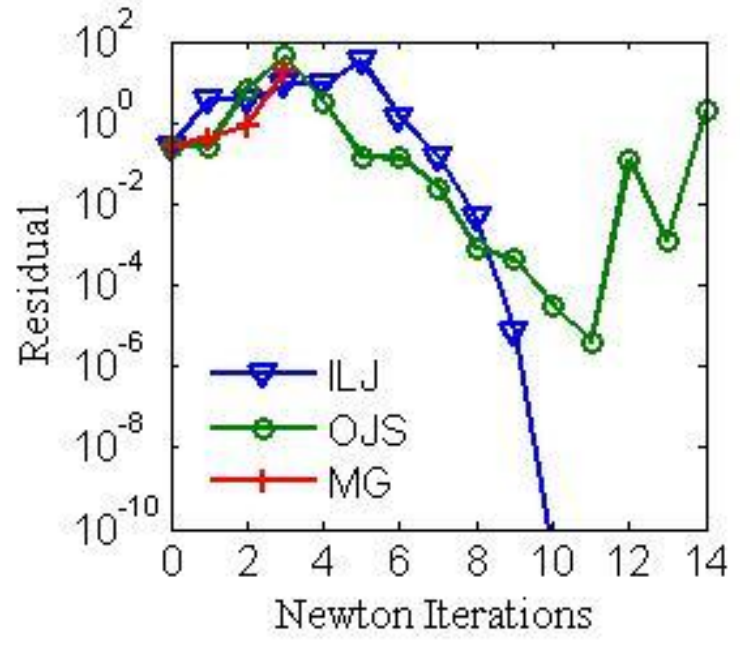

Figure 17. Convergence behavior for ILJ, OJS and MG three-phase flash calculations at 823 psi and $57^{\circ} \mathrm{F}$. Only, ILJ converged to a nontrivial physical solution.

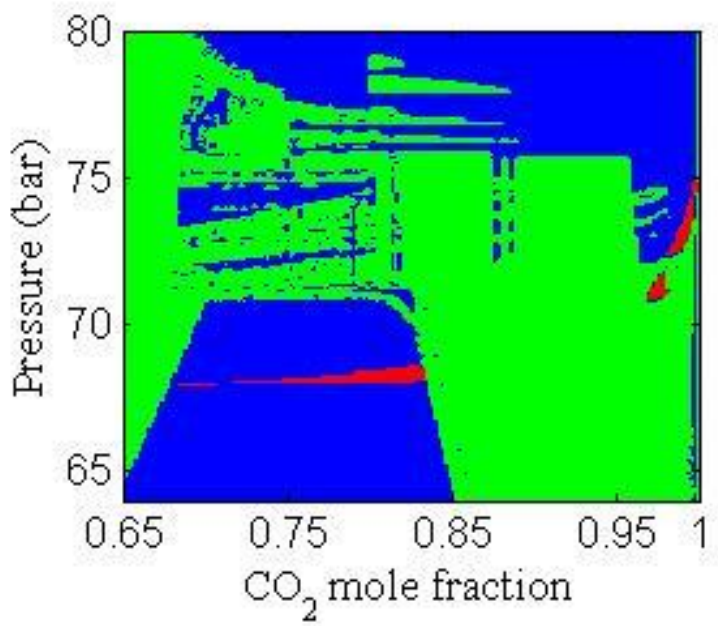

Figure 19. Number of phases found for Maljamar separator oil at $305.35 \mathrm{~K}$ by using only one SS iteration and a maximum of 20 MG Newton iterations. Red, blue and green regions converged to three-phase, two-phase and single-phase regions, respectively. MG convergence is poor.

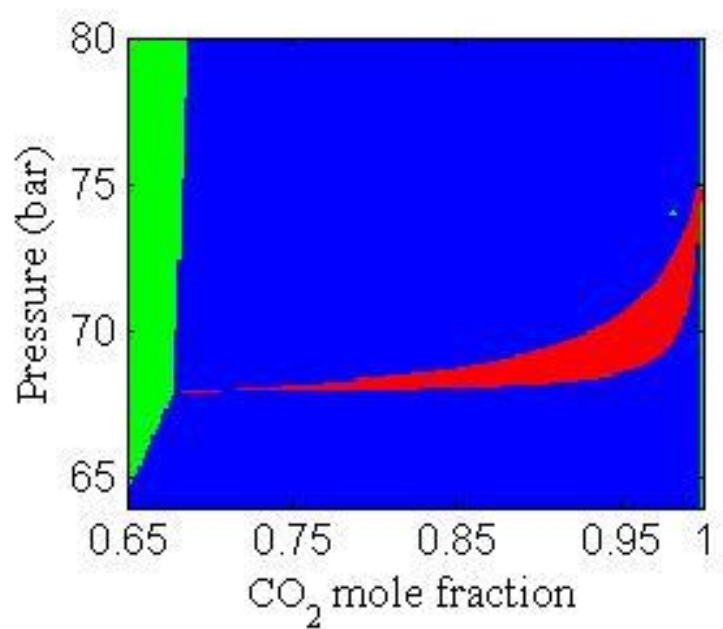

Figure 18. Number of phases for Maljamar separator oil at $305.35 \mathrm{~K}$ by using SS iterations only. Maximum number of iterations is 1000000 . Red, blue and green regions converged to three-phase, two-phase and single-phase regions, respectively. This is the taken as the htruth model for this fluid.

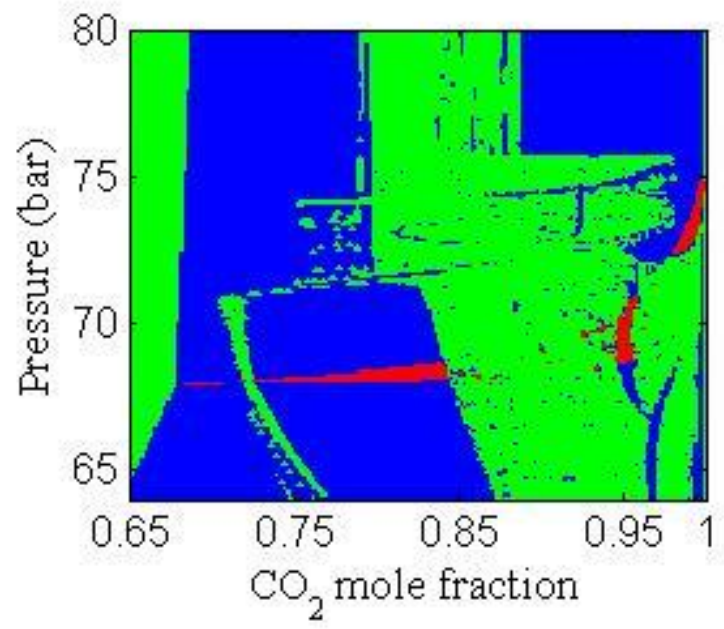

Figure 20. Number of phases found for Maljamar separator oil at $305.35 \mathrm{~K}$ by using only one SS iteration and a maximum of 20 OJS Newton iterations. Red, blue and green regions converged to three-phase, two-phase and single-phase regions, respectively. OJS convergence is improved over MG in Fig. 19. 


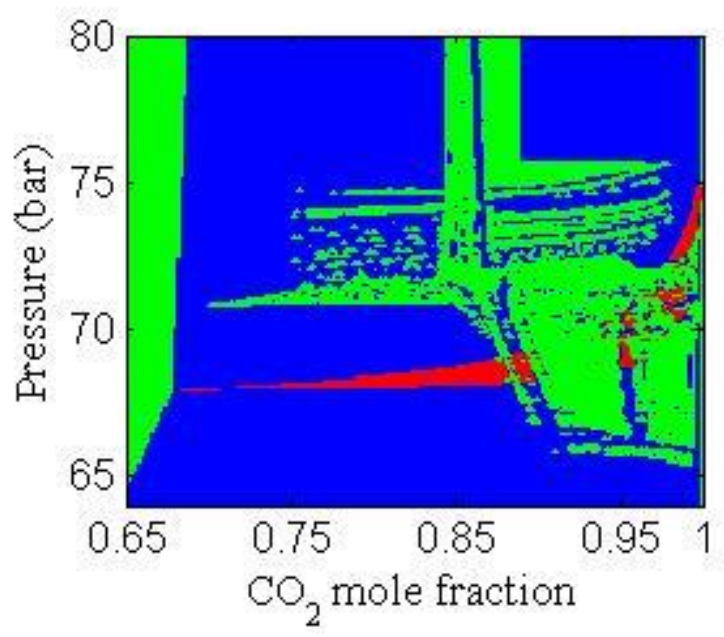

Figure 21. Number of phases found for Maljamar separator oil at $305.35 \mathrm{~K}$ using only one SS iteration and a maximum of 20 ILJ Newton iterations. Red, blue and green regions converged to three-phase, twophase and single-phase regions, respectively. ILJ gives the best convergence.

\section{Nomenclature}

A Attraction parameter

$B \quad$ Repulsion parameter

$e \quad$ ILJ residual function

$J \quad$ Jacobian matrix

$N_{C} \quad$ Number of components

$N_{p} \quad$ Number of phases

Z Compressibility factor

$f \quad$ Fugacity

$h_{i} \quad$ LJ vector

$h \quad$ Independent variables for improved algorithms

$G \quad$ Dimensionless molar Gibbs energy

$g_{i} \quad$ LJ vector, gradient vector

$K_{i} \quad \mathrm{~K}$-value

$\mathrm{k}_{\mathrm{ij}} \quad$ BIP matrix

$n \quad$ Number of eigenvalues considered; iteration number

$q \quad$ Eigenvector

$q_{i \alpha} \quad$ Constant matrix of improved reduced methods

$R \quad$ Residual function, convergence criterion

$S \quad$ Stability analysis residual function

$X \quad$ Stationary point

$x \quad$ Liquid molar composition

$y \quad$ Vapor composition

$z \quad$ Overall composition

\section{Greek letters}

$\delta_{1}, \delta_{2} \quad$ EOS parameters

$\phi \quad$ Fugacity coefficient

$\Theta \quad$ LJ reduced variables

$\lambda \quad$ Eigenvalue

$\alpha \quad$ Reduced variable index

$\beta \quad$ Phase mole fraction

$\psi_{k} \quad$ Set of ILJ variables

$\varepsilon \quad$ Convergence/Switch tolerance

\section{Subscripts}

$i \quad$ Composition

$j \quad$ Composition or phase

$L \quad$ Liquid

V Vapor

\section{Abbreviations}

BIP Binary interaction parameters

ILJ Our new algorithm based on Gorucu and Johns (2014)

MG Conventional method based on minimization of Gibbs energy

NR Newton-Raphson

NWE North Ward Estes

OJS Algorithm based on Okuno, Johns and Sepehrnoori (2010a)

SS Successive substitution

TPD Tangent plane distance 


\section{Acknowledgments}

This research was conducted with the support of the Gas Flooding Research Project at the EMS Energy Institute at the Pennsylvania State University. Dr. Russell T. Johns is the Beghini Professor of Petroleum and Natural Gas Engineering in the John and Willie Leone Family Department of Energy and Mineral Engineering at the Pennsylvania State University. Seyhan Emre Gorucu is a reservoir simulation scientist at Computer Modelling Group.

\section{References}

Baker, L. E., Pierce, A. C., and Luks, K. D. Gibbs Energy Analysis of Phase Equilibria. SPE Journal 22 (5): 731-742 (1982).

Gorucu, S. E. PhD Dissertation. Reduced Phase Equilibrium Calculations: New Reduced Parameters, Critical Analysis and Fluid Characterization. The Pennsylvania State University, December 2013.

Gorucu, S. E., and Johns, R. T. New Reduced Parameters for Flash Calculations Based on Two-Parameter BIP Formula. Journal of Petroleum and Science Engineering, 116: 50-68 (2014).

Gorucu, S. E., and Johns, R. T. Comparison of Reduced and Conventional Two-Phase Flash Calculations. SPE Journal. 20 (2): 294-305 (2015).

Haugen, K.B., and Beckner B.L. A Critical Comparison of Reduced and Conventional EOS Algorithms. SPE Journal. 18 (2): 378-388 (2013).

Hendriks, E.M. Reduction Theorem for Phase Equilibrium Problems. Ind. Eng. Chem. Res. 27 (9): 1728-1732 (1988).
Hendriks, E.M., and van Bergen, A.R.D. Application of a Reduction Method to Phase Equilibria Calculations. Fluid Phase Equilibria 74: 17-34 (1992).

Hoteit, H. and Firoozabadi F. Simple Phase Stability-Testing Algorithm in the Reduction Method. AlChe J. 52(8): 2909-2920 (2006).

Jensen, B.H., and Fredenslund, A. A Simplified Flash Procedure for Multicomponent Mixtures Containing Hydrocarbons and one NonHydrocarbon Using Two-Parameter Cubic Equations of State. Ind. Eng. Chem. Res. 26 (10): 2129-2134 (1987).

Khan, S. A., Pope, G. A. and Sepehrnoori, K. Fluid Characterization of Three-Phase $\mathrm{CO}_{2} / \mathrm{Oil}$ Mixtures. SPE/DOE Eight Symposium on Enhanced Oil Recovery (253-265). Tulsa (1992).

Li, Y., and Johns, R.T. Rapid Flash Calculations for Compositional Simulation. SPE Reservoir Evaluation and Engineering 9 (5): 521-529 (2006).

Li, H., Zheng, S and Yang, D. Enhanced Swelling Effect and Viscosity Reduction of Solvent(s)/CO2/Heavy-Oil Systems. SPE Journal 18 (4): 695-707 (2013).

Li, Z., and Firoozabadi, A. General Strategy for Stability Testing and Phase-Split Calculations in Two and Three Phases. SPE Journal 17 (4): 1096-1107 (2012).

Michelsen, M.L. Simplified Flash Calculations for Cubic Equations of State. Industrial and Engineering Chemistry Process Design and Development 25 (1): 184-188 (1986).

Michelsen, M. L., and Mollerup, J. M. Thermodynamic Models: Fundamentals \& Computational Aspects (2 ed.). Holte: Tie-Line Publications, 2007.

Mohebbinia, S., Sepehrnoori, K., and Johns, R. T. Four-Phase Equilibrium Calculations of Carbon Dioxide/Hydrocarbon/Water Systems 
With a Reduced Method. SPE Journal 18 (5): 943-951 (2013).

Mohebbinia, S., Sepehrnoori, K., Johns, R. T. and Korrani A. K. N. Simulation of Asphaltene Precipitation during Gas Injection Using PCSAFT EOS, 2014. Presented at the SPE Annual Technical Conference and Exhibition, Amsterdam.

Nichita, D. V., Broseta. D., and De Hemptinne, J-. C. Multiphase Equilibrium Calculation Using Reduced Variables. Fluid Phase Equilibria 246 (1-2): 15-27 (2006).

Nichita, D.V., and Graciaa, A. A New Reduction Method for Phase Equilibrium Calculations. Fluid Phase Equilibria 302 (1-2): 226-233 (2010).

Nichita, V. and Petitfrere, M. Phase stability analysis using a reduction method. Fluid Phase Equilibria, 358: 27-39 (2013).

Okuno, R. Modeling of Multiphase Behavior for Gas Flooding Simulation, $\mathrm{PhD}$ dissertation, 2009.

Okuno, R., Johns, R.T., and Sepehrnoori, K. Application of a Reduced Method in Compositional Simulation. SPE Journal 15 (1): 39-49 (2010a).

Okuno, R., Johns, R. T., \& Sepehrnoori, K. Three-Phase Flash in Compositional Simulation Using a Reduced Method. SPE Journal 15 (1): 689-703 (2010b).

Okuno, R., Johns, R. T., and Sepehrnoori, K. (2010c). A New Algorithm for Rachford-Rice for Multiphase Compositional Simulation. SPE Journal 15 (2): 313-325 (2010c).

Orr, F. M., Yu, A. D., \& Lien, C. L. Phase Behavior of $\mathrm{CO} 2$ and Crude Oil in LowTemperature Reservoirs. SPE Journal 21(4): 480-492 (1981).

Pan, H. and Firoozabadi, A. Fast and Robust Algorithm for Compositional Modeling: Part I Stability Analysis Testing. SPE Journal 7 (1): 78-89 (2002).

Peng, D. Y. and Robinson, D. B. The Characterization of the Heptanes and Heavier Fractions for the GPA Peng-Robinson Programs. Gas Processor Association (1978).

Petitfrere, M. and Nichita, D. V. A Comparison of Conventional and Reduction Approaches for Phase Equilibrium Calculations. Fluid Phase Equilibria 386: 30-46 (2015a).

Petitfrere, M. and Nichita, D. V. Multiphase Equilibrium Calculations Using a Reduction Method. Fluid Phase Equilibria 401: 110-126 (2015b).

Perschke, D. R., Chang, Y. -B., Pope, G. and Sepehrnoori, K. (1989). Comparison of Phase Behavior Algorithms for an Equation of State Compositional Simulator. SPE-19443-MS.

Turek, E. A., Metcalfe, R. S., Fishback, R. E. Phase Behavior of Several CO2/ West TexasReservoir-Oil Systems. SPE Reservoir Engineering 3 (2): 505-516 (1988).

Wilson, G., A Modified Redlich-Kwong Equation of State. Application to General Physical Data Calculations, 1969. Presented at AlChe $65^{\text {th }}$ National Meeting, Cleveland, Ohio. 


\section{Appendix}

In this appendix we derive the equations for the construction of the Jacobian matrix using our reduced method. The Jacobian matrix for the new three-phase flash calculations is given by the equations,

$$
\begin{array}{ll}
\frac{\partial e_{\alpha}}{\partial \psi_{k}}=\frac{\partial h_{\alpha}^{3}}{\partial \psi_{k}}-\frac{\partial h_{\alpha}^{1}}{\partial \psi_{k}}-\delta_{\alpha k} & \alpha=1, \ldots, 6 \\
\frac{\partial e_{\alpha}}{\partial \psi_{k}}=\frac{\partial h_{\alpha}^{3}}{\partial \psi_{k}}-\frac{\partial h_{\alpha}^{2}}{\partial \psi_{k}}-\delta_{\alpha k} & \alpha=7, \ldots, 12
\end{array}
$$

Thus, the Jacobian matrix can be illustrated as follows.

$$
\left[\begin{array}{ccc}
\frac{\partial h_{\alpha}^{3}}{\partial \Delta h_{k}^{13}}-\frac{\partial h_{\alpha}^{1}}{\partial \Delta h_{k}^{13}}-\delta_{\alpha k} & \vdots & \frac{\partial h_{\alpha}^{3}}{\partial \Delta h_{k}^{23}}-\frac{\partial h_{\alpha}^{1}}{\partial \Delta h_{k}^{23}} \\
\cdots & \vdots & \cdots \\
\frac{\partial h_{\alpha}^{3}}{\partial \Delta h_{k}^{13}}-\frac{\partial h_{\alpha}^{2}}{\partial \Delta h_{k}^{13}} & \vdots & \frac{\partial h_{\alpha}^{3}}{\partial \Delta h_{k}^{23}}-\frac{\partial h_{\alpha}^{2}}{\partial \Delta h_{k}^{23}}-\delta_{\alpha k}
\end{array}\right]
$$

The derived equations are similar to those of Nichita and Graciaa (2011) and Okuno (2009). The analytical derivatives are

$$
\frac{\partial h_{\alpha j}}{\partial \Delta h_{\beta}}=\sum_{\gamma=1}^{5} \frac{\partial h_{\alpha j}}{\partial Q_{\gamma j}} \frac{\partial Q_{\gamma j}}{\partial \Delta h_{\beta}} \quad \alpha, \beta=1, \ldots 6 ; \quad \gamma=1, \ldots, 5 \quad \text { and } \mathrm{j}=1, \ldots, 3
$$

where $\partial h_{\alpha j} / \partial Q_{\gamma j}$ is the same as for the two-phase flash calculations. Analytical expressions for $\partial Q_{\gamma j} / \partial \Delta h_{\beta}$ are different, however, and are shown below.

$$
\frac{\partial Q_{\gamma j}}{\partial \Delta h_{\beta}}=\sum_{i=1}^{N_{c}} \theta_{i \gamma} \frac{\partial x_{i}^{j}}{\partial \Delta h_{\beta}} \quad \text { where } \quad h_{\beta}=\Delta h_{k}^{13} \text { or } \Delta h_{k}^{23}
$$

A material balance gives,

$$
x_{i}^{1}=\frac{K_{i 1} z_{i}}{t_{i}}, \quad x_{i}^{2}=\frac{K_{i 2} z_{i}}{t_{i}} \quad, \quad x_{i}^{3}=\frac{z_{i}}{t_{i}}
$$


where $t_{i}=K_{i 1} \beta_{1}+K_{i 2} \beta_{2}+\beta_{3}, K_{i 1}=\phi_{i 3} / \phi_{i 1}, K_{i 2}=\phi_{i 3} / \phi_{i 2}$. The parameters $x_{i}^{1}, x_{i}^{2}$ and $x_{i}^{3}$ are phase compositions, respectively. The parameter $\beta$ is phase mole fraction, $i$ is component index, and $j$ is phase index. Therefore, the following can be derived:

$$
\begin{gathered}
\frac{\partial x_{i}^{1}}{\partial \Delta h_{k}^{13}}=\frac{z_{i}}{\left(t_{i}\right)^{2}}\left(\frac{\partial K_{i 1}}{\partial \Delta h_{k}^{13}}\left[K_{i 2} \beta_{2}+\beta_{3}\right]-K_{i 1}\left(\frac{\partial \beta_{1}}{\partial \Delta h_{k}^{13}}\left(K_{i 1}-1\right)+\frac{\partial \beta_{2}}{\partial \Delta h_{k}^{13}}\left(K_{i 2}-1\right)\right)\right) \\
\frac{\partial x_{i}^{1}}{\partial \Delta h_{k}^{23}}=-\frac{K_{i 1} z_{i}}{\left(t_{i}\right)^{2}}\left(\frac{\partial \beta_{1}}{\partial \Delta h_{k}^{23}}\left(K_{i 1}-1\right)+\beta_{2} \frac{\partial K_{i 2}}{\partial \Delta h_{k}^{23}}+\frac{\partial \beta_{2}}{\partial \Delta h_{k}^{23}}\left(K_{i 2}-1\right)\right) \\
\frac{\partial x_{i}^{2}}{\partial \Delta h_{k}^{13}}=-\frac{K_{i 2} z_{i}}{\left(t_{i}\right)^{2}}\left(\beta_{1} \frac{\partial K_{i 1}}{\partial \Delta h_{k}^{13}}+\frac{\partial \beta_{1}}{\partial \Delta h_{k}^{13}}\left(K_{i 1}-1\right)+\frac{\partial \beta_{2}}{\partial \Delta h_{k}^{13}}\left(K_{i 2}-1\right)\right) \\
\frac{\partial x_{i}^{2}}{\partial \Delta h_{k}^{23}}=\frac{z_{i}}{\left(t_{i}\right)^{2}}\left(\frac{\partial K_{i 2}}{\partial \Delta h_{k}^{23}}\left[K_{i 1} \beta_{1}+\beta_{3}\right]-K_{i 2}\left(\frac{\partial \beta_{1}}{\partial \Delta h_{k}^{23}}\left(K_{i 1}-1\right)+\frac{\partial \beta_{2}}{\partial \Delta h_{k}^{23}}\left(K_{i 2}-1\right)\right)\right) \\
\frac{\partial x_{i}^{3}}{\partial \Delta h_{k}^{13}}=-\frac{z_{i}}{\left(t_{i}\right)^{2}}\left(\beta_{1} \frac{\partial K_{i 1}}{\partial \Delta h_{k}^{13}}+\frac{\partial \beta_{1}}{\partial \Delta h_{k}^{13}}\left(K_{i 1}-1\right)+\frac{\partial \beta_{2}}{\partial \Delta h_{k}^{13}}\left(K_{i 2}-1\right)\right) \\
\frac{\partial x_{i}^{3}}{\partial \Delta h_{k}^{23}}=-\frac{z_{i}}{\left(t_{i}\right)^{2}}\left(\beta_{2} \frac{\partial K_{i 2}}{\partial \Delta h_{k}^{23}}+\frac{\partial \beta_{1}}{\partial \Delta h_{k}^{23}}\left(K_{i 1}-1\right)+\frac{\partial \beta_{2}}{\partial \Delta h_{k}^{23}}\left(K_{i 2}-1\right)\right) .
\end{gathered}
$$

The material balance yields

$$
\sum_{i=1}^{N_{C}} \frac{z_{i}\left(K_{i 1}-1\right)}{K_{i 1} \beta_{1}+K_{i 2} \beta_{2}+\beta_{3}}=0
$$

Substitution of $\beta_{1}+\beta_{2}+\beta_{3}=1, \partial K_{i 2} / \partial \Delta h_{13}=0$ and $\partial \beta_{3} / \partial \Delta h_{13}=-\partial \beta_{1} / \partial \Delta h_{13}-\partial \beta_{2} / \partial \Delta h_{13}$ into the above equation gives,

$$
\begin{gathered}
\frac{\partial \beta_{1}}{\partial \Delta h_{13}}=\frac{c \sum_{i=1}^{N_{C}}\left(d_{i}\left(K_{i 2}-1\right) \frac{\partial K_{i 1}}{\partial \Delta h_{13}} \beta_{1}\right)+b \sum_{i=1}^{N_{C}} d_{i} \frac{\partial K_{i 1}}{\partial \Delta h_{13}}\left(K_{i 2} \beta_{2}-\beta_{2}+1\right)}{a b-c^{2}} \\
\frac{\partial \beta_{1}}{\partial \Delta h_{23}}=\frac{-c \sum_{i=1}^{N_{C}}\left(d_{i}\left(K_{i 1} \beta_{1}-\beta_{1}+1\right) \frac{\partial K_{i 12}}{\partial \Delta h_{23}} \beta_{1}\right)-b \sum_{i=1}^{N_{C}}\left(d_{i} \frac{\partial K_{i 1}}{\partial \Delta h_{23}}\left(K_{i 1}-1\right) \beta_{2}\right)}{a b-c^{2}}
\end{gathered}
$$


where $a=\sum_{i=1}^{N_{C}} d_{i}\left(K_{i 1}-1\right)^{2}, \quad b=\sum_{i=1}^{N_{C}} d_{i}\left(K_{i 2}-1\right)^{2}, \quad$ and $\quad c=\sum_{i=1}^{N_{C}} d_{i}\left(K_{i 2}-1\right)\left(K_{i 1}-1\right) . \quad$ The derivatives $\partial \beta_{2} / \partial \Delta h_{13}, \partial \beta_{2} / \partial \Delta h_{23}, \partial \beta_{3} / \partial \Delta h_{13}, \partial \beta_{3} / \partial \Delta h_{23}$ are easily found after calculation of $\partial \beta_{1} / \partial \Delta h_{13}$ and $\partial \beta_{1} / \partial \Delta h_{23}$. 